Anderson, E. S. \& Fraser, A. (1955). J. gen. Microbiol. 13, 519-532

\title{
The Influence of the Factors Determining Vi-type Specificity in Salmonella typhi on the Adaptation of Vi-phage II
}

\author{
By E. S. ANDERSON AND ANTHEA FRASER \\ Central Enteric Reference Laboratory and Bureau, Public Health Laboratory \\ Service (Medical Research Council), London, N.W.9
}

\begin{abstract}
SUMMARY: All adaptations of Vi-phage II to the known Vi-types of Salmonella typhi have been grown on Vi-type A. In confirmation of earlier work these adaptations were found to be phenotypic or genotypic modifications of the original Viphage II, or showed a combination of both changes. Most adaptations to non-lysogenic types are changes of phenotype only, whereas those to lysogenic types are usually genotypically stable, although they may have a superimposed phenotypic modification. The fact that Vi-types that are exactly similar to naturally occurring types can be made artificially by the lysogenization of non-lysogenic types with type-determining phages makes it possible to assign structural formulae to most of the known naturally occurring Vi-types. The formulae consist of the symbol of the non-lysogenic precursor of a lysogenic type followed by that of the type-determining temperate phage in parenthesis. Thus, type F2 is type F1 carrying phage f2 and the formula of type F2 is therefore F1(f2). Using these formulae it is possible to predict the host range of Vi-phage II adapted to each lysogenic Vi-type. The phenotypic and genotypic changes concerned in the many adaptations of Vi-phage II are independent of one another, and by taking advantage of this independence it is possible to adduce experimental proof of the correctness of the proposed structural formulae for the lysogenically determined Vi-types. In studying the Vi-types it is useful to regroup those that are lysogenic according to the non-lysogenic precursor types common to certain of them.
\end{abstract}

The Vi-phage typing of Salmonella typhi (Craigie \& Yen, 1938) is now recognized to be one of the most valuable guides to the epidemiology of typhoid fever. There are at present 33 recognized Vi-types of the typhoid bacillus. The convention, originated by Craigie and Yen, of designating Vi-types and their corresponding adapted phages by identical symbols is still maintained. The earlier types and their adapted phages were designated by capital letters, but most of those more recently identified have been assigned numerical symbols. The reactions of the internationally recognized Vi-types with the adapted phage preparations in routine test dilution* are shown in Table 1. As this Table shows, type $A$ is sensitive to all the typing preparations, irrespective of their specificity.

The parent phage of the adapted typing preparations was named by Craigie \& Yen (1938) Vi-phage II : it is specific for the Vi-positive form of the typhoid bacillus, and is not adsorbed by the Vi-negative form because of the absence of the $\mathrm{Vi}$ antigen which is necessary for its attachment to the bacterial cell. All

* The routine test dilution of a phage is that giving confluent or semi-confluent lysis on the homologous type and a minimum of cross-reactions on heterologous types. 
Vi-types of Salmonella typhi will adsorb all the adapted Vi-typing preparations, irrespective of their specificity (Craigie, 1940), but lysis only occurs when the type specificities of the organism and the phage coincide. A lethal effect on the bacteria is apparent when a culture of $\boldsymbol{S}$. typhi adsorbs a heterologous Vityping preparation. This is especially marked when the phage multiplicity, that is, the phage/organism ratio, is high (Anderson \& Fraser, unpublished). All the Vi-typing phage preparations are neutralized by anti-Vi-phage II serum prepared against phage $A$.

The factors which control Vi-type specificity in Salmonella typhi have been discussed in previous publications (Anderson, 1951; Felix \& Anderson, 1951; Anderson \& Felix, 1953b). It has been shown that Vi-type specificity in the typhoid bacillus is frequently an expression of a pattern of resistance of the organism to the typing phages which is dependent on lysogenicity of the particular types concerned. Of the $33 \mathrm{Vi}$-types recognized at present, 11 owe their specificity to this cause. The symbiotic or temperate phages responsible for Vi-type specificity are called type-determining phages. They are $\mathbf{O}$ phages and will attack and lysogenize non-Vi forms of the typhoid bacillus. In addition to controlling the reactions of strains of the typhoid bacillus with the Vi-typing phages, the type-determining phages also affect their sensitivity to $O$ phages. Although the Vi antigen acts as the primary site of attachment of Vi-phage II to the typhoid bacillus, therefore, type specificity of which lysis by the adapted Vi-phages is an expression would appear to be determined elsewhere in the soma of the bacterial cell.

The type-determining phages are designated by small letters corresponding to the capitals by which the Vi-types concerned are known, and by a number with a superscript prime sign when the lysogenic Vi-type has a numerical designation. For example, type D 1 carries phage $d 1$, and type D 6 phage $d 6$; type 25 carries phage $25^{\prime}$, and type 26 phage $26^{\prime}$. Some types carry phages indistinguishable from those found in other types, in which case the phages are assigned an identical symbol. Thus, the determining phage of type $\mathbf{3 3}$ is indistinguishable from that of type $\mathrm{D} 6$ and the phage carried by both types is therefore called $\mathrm{d} 6$.

By lysogenizing selected non-lysogenic types with type-determining phages, Vi-types can be artificially prepared that appear to be identical with those occurring naturally. All but one (the exception is type $\mathrm{D} 4$ ) of the lysogenically determined types hitherto encountered in nature have now been prepared artificially in this way. In addition, several types not yet encountered in the field have been synthesized and it is possible to adapt Vi-phage II to most of these artificial new types to produce typing phages, usually of high specificity and reasonable working titre.

The alterations in host range that occur during the adaptation of Vi-phage II to the Vi-types of Salmonella typhi to produce the specific typing phages are in many cases caused by a change of phenotype only (' host-induced modification' of Luria \& Human, 1952). The proof of the non-mutational character of this change lies in the results of propagation of the phages concerned on type $\mathbf{A}$ : every particle of a phenotypically modified preparation changes to phage $\mathbf{A}$. 


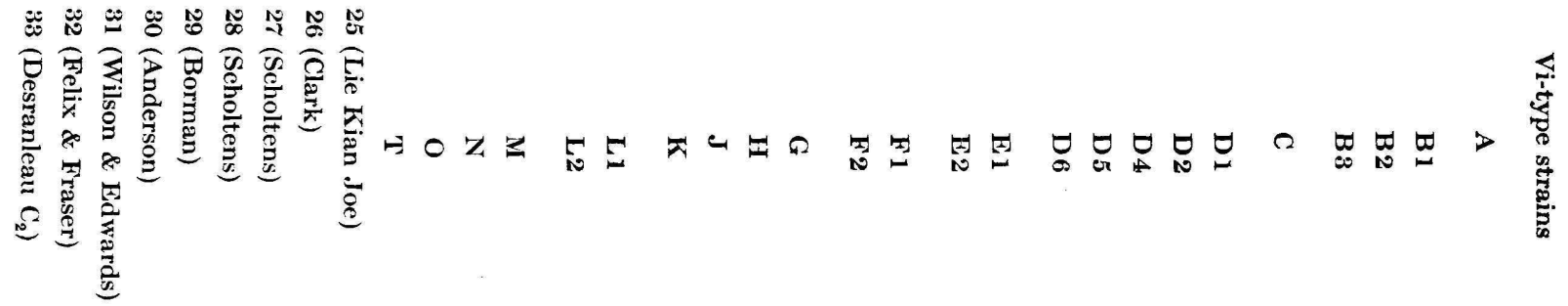

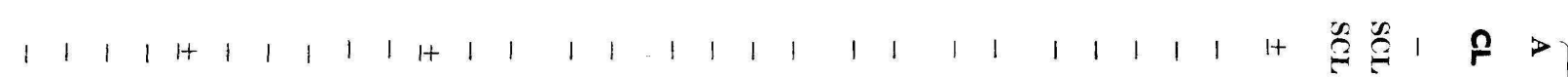

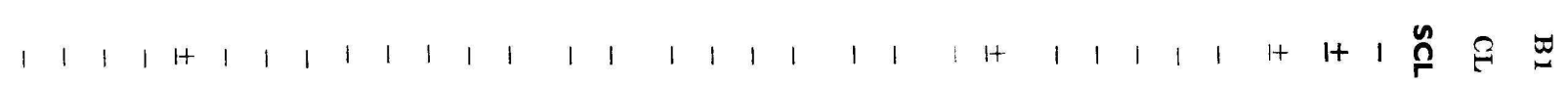

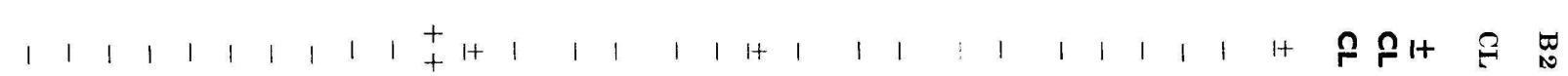

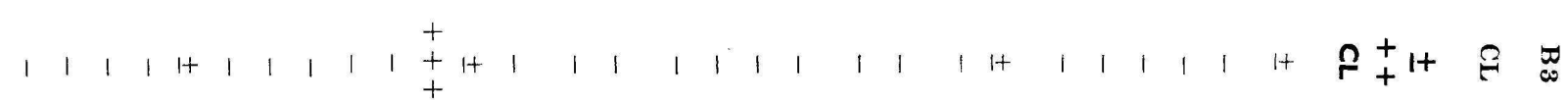

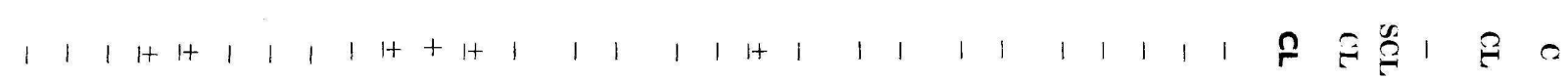

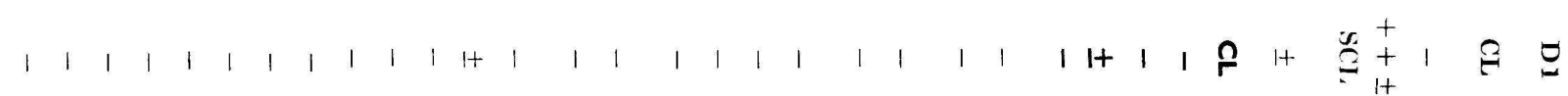

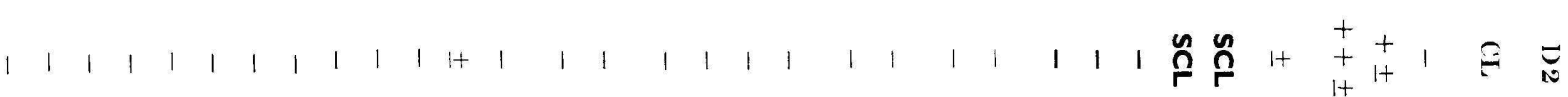

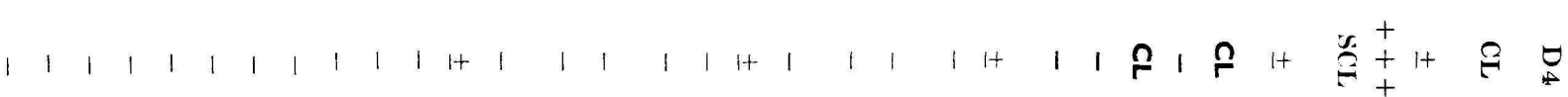

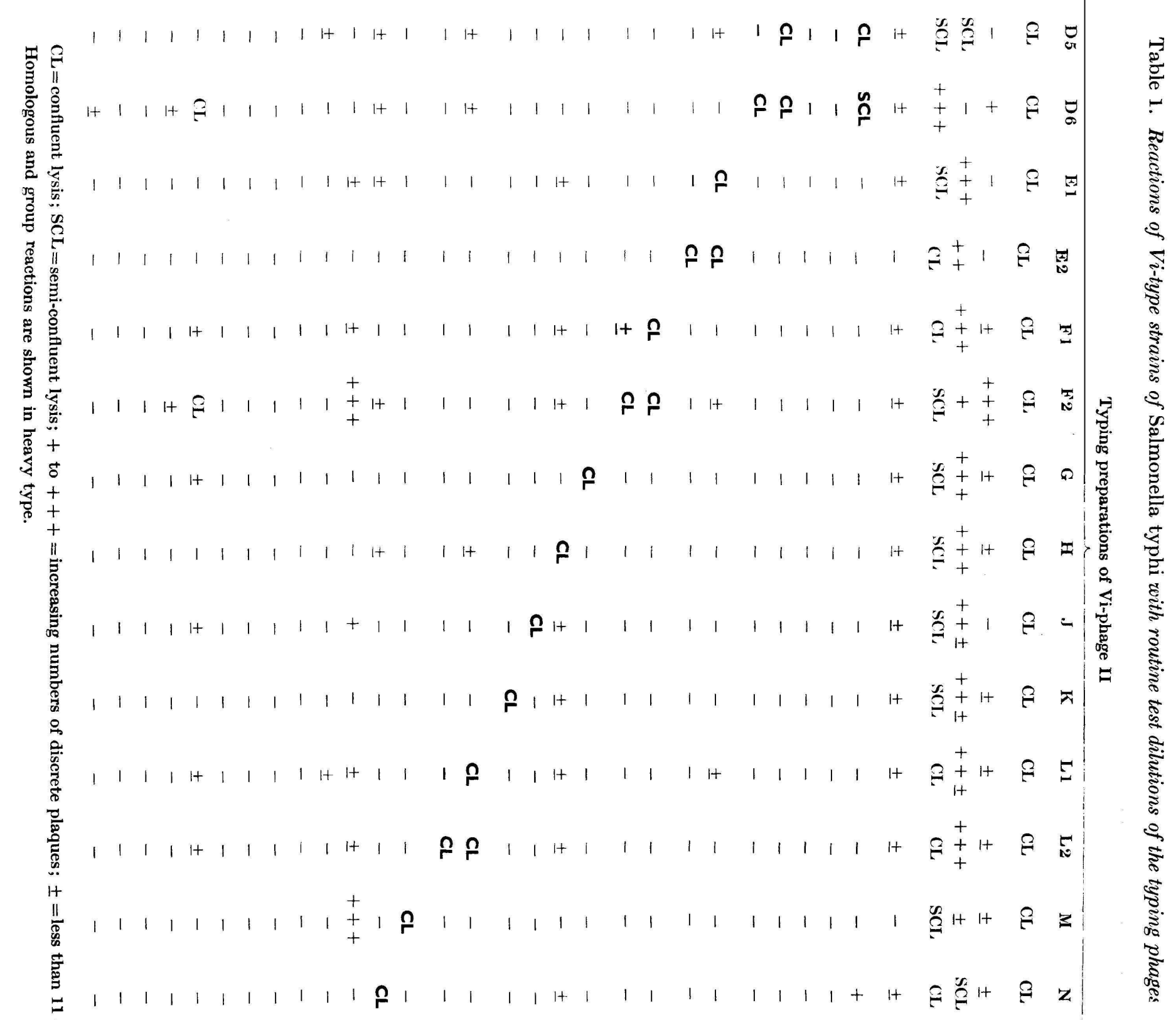

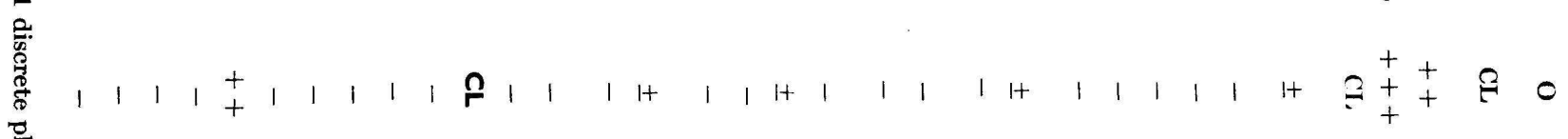

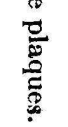

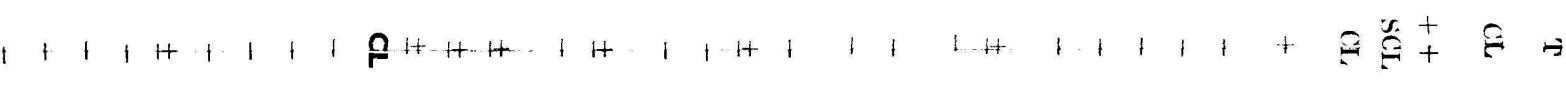

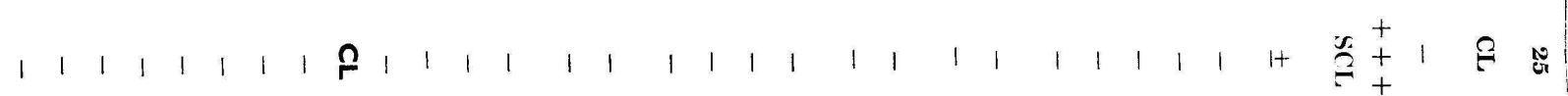

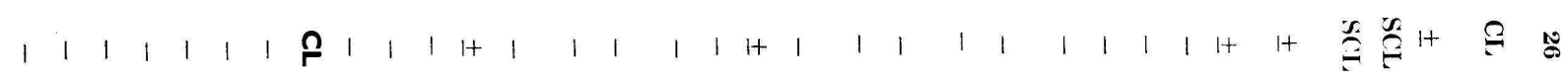

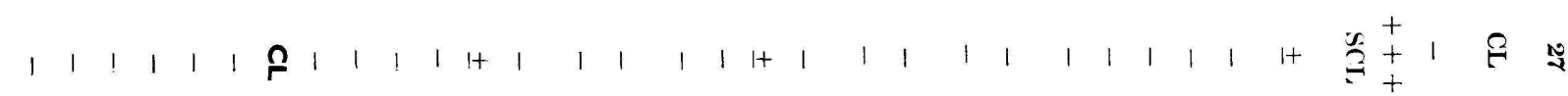

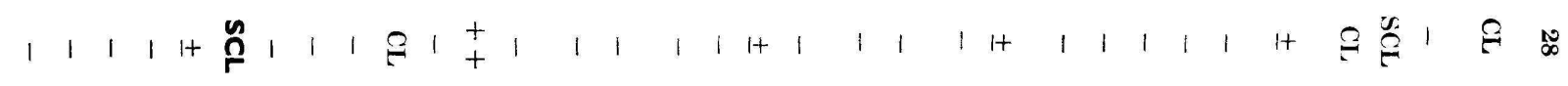

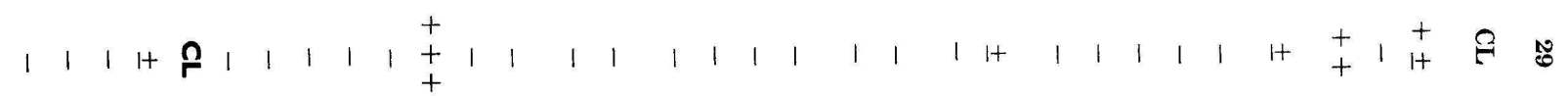

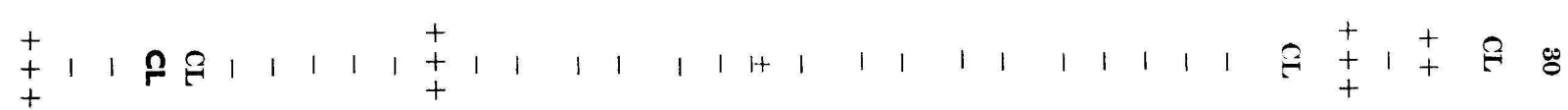

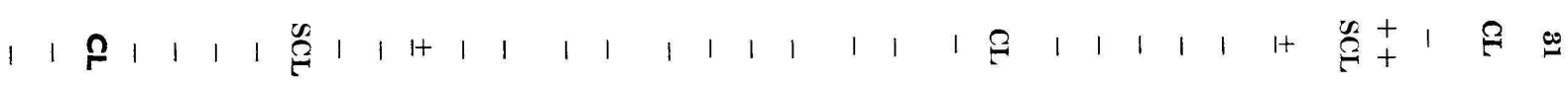

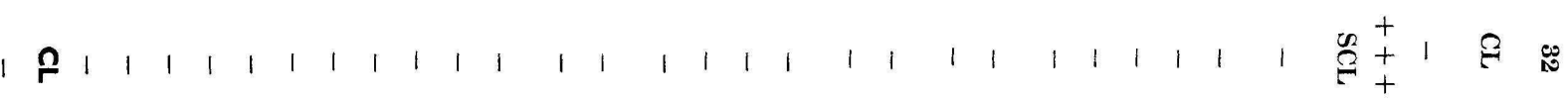

$$
\begin{aligned}
& \text { O }
\end{aligned}
$$

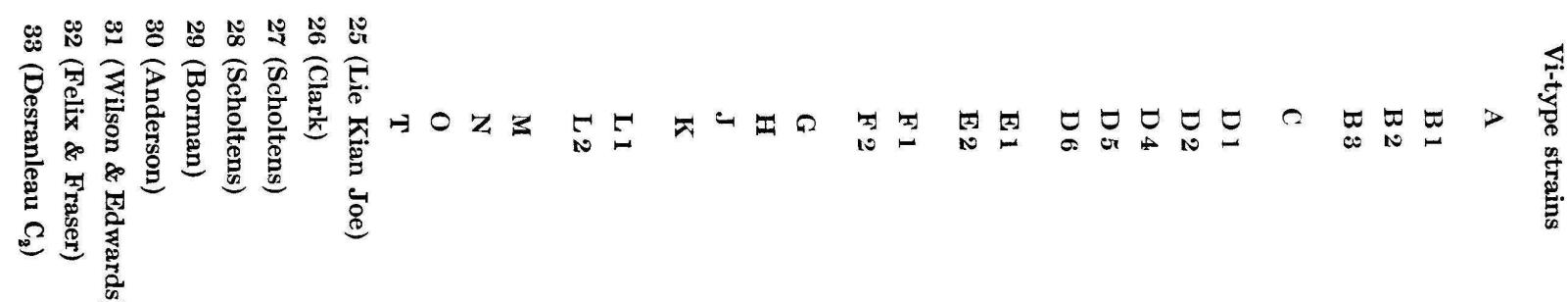


Some 'adapted' phages, however, are true host-range mutants and these may also undergo modifications of phenotype. Thus, the adapted Vi-typing preparations may owe their various host ranges to the following combinations: wild genotype, modified phenotype; modified genotype, wild phenotype; modified genotype, modified phenotype. Phage $\mathbf{A}$, which has the narrowest host range of all the Vi-typing phages and seems to be the most primitive form of Vi-phage II, can be regarded as wild genotype, wild phenotype. Obviously, a host-range mutant descended from phage $\mathrm{A}$, since it lyses other types than type $\mathbf{A}$, is not of the same phenotype as phage $\mathbf{A}$. However, as it has been found that changes in phenotype of the phage can occur without mutation, the convention has been adopted of referring to the preparations that have undergone this non-hereditary change as being phenotypically modified, and the phages that have undergone mutation only are described as being genotypically modified or as host-range mutants, with the implication that they retain the wild phenotype in the portion of the phage that is subject to host-induced modification. Host-range mutants that have undergone host-induced modification are described as phenotypically modified mutants.

Anderson \& Felix $(1952,1953 a, c)$ propagated a number of adapted Vityping preparations on type $\mathbf{A}$ and determined the host range of the phages so obtained. Ten phages were investigated : five changed to phage $A$, that is, they were phenotypic modifications as defined above; four were propagated unchanged (genotypic modifications or host-range mutants); and one changed its host range without becoming phage $\mathbf{A}$ (phenotypically modified mutant).

\section{METHODS}

Media. The nutrient basis of the media used in these experiments was Bacto dehydrated nutrient broth (Difco Laboratories). This was used in a strength of $2 \%$, with the addition of $0.85 \% \mathrm{NaCl}$. The base for solid media was $1 \cdot 3 \%$ New Zealand powdered agar. These media are referred to as 'Difco' broth and 'Difco' agar.

Titration of phages. Phages were titrated by the 'spot titration' method, serial dilutions being spotted with a standard loop of $\mathbf{3} \mathrm{mm}$. internal diameter on to a freshly spread lawn of organisms of the various Vi-types under test on Difco agar plates. These loops deliver a volume very close to $\mathbf{0 . 0 1} \mathrm{ml}$.

Preparation and identification of phages. The new phage preparations were grown as follows:

A lawn of logarithmically growing organisms of the type on which the phage was to be propagated was spread on the surface of Difco agar plates and allowed to dry. The phage under examination was applied to this culture in decimal dilutions. The plates were incubated overnight, and the following morning three to six discrete plaques, together with a small amount of surrounding culture, were cut from suitable dilutions. Each plaque was transferred to $20 \mathrm{ml}$. of Difco broth in a screw-capped bottle and incubated at $38.5^{\circ}$ until no further lysis appeared to be taking place. The host cells were then killed by heating the bottles at $57^{\circ}$ for 40 min., after which the lysates were centrifuged to remove the dead bacteria. 
Phages were designated as fractions, the numerator indicating the starting phage, and the denominator the host strain on which it had grown. Thus, phage 26/E 1 resulted from the growth of phage 26 on type E1. All the adapted phages described in the following pages were descended from single plaques. Three to six phage lines were grown in each experiment and most experiments were confirmed by repetition.

The type specificity of newly grown phages was identified by titrating them on a range of Vi-type strains. Type $A$, which is sensitive to all Vi-typing phages, was included in all titrations. The additional types used were: that on which the phage had formerly been grown to produce the specificity of which the stability was under examination; that on which the phage had most recently been grown; one or more related types if available that might be sensitive to the early or later adaptations; and a few types chosen at random that would be expected to be resistant to the former and the latest adaptations. For instance, phage 26/E 1 was tested on types A, 26 (former adaptation), E1 (latest adaptation), 31 (related to types 26 and $\mathrm{E} 1$ (see Table 1) and expected to be lysed by the newly grown phage) and C and F1 (resistant to phages 26 and 31). Thus, by the use of a limited number of Vi-types, it was possible to establish the specificity of the new phages.

\section{RESULTS}

Table 2 shows the result of growing phage $\mathbf{L} 2$ on type $A$. This titration is given in full in order to indicate the range of Vi-types on which the phages were tested. A titration of phage L 2 on the same range of types is given for purposes of comparison. The single-plaque phages in Table 2 had been cut from this titration of phage L 2 on type A. It can be seen from Table 2 that phage L 2, which normally attacks types A, L1 and L 2 with equal readiness, had lost its ability to lyse the latter two types, had apparently not acquired new host ranges, and had retained only the capacity to attack type $A$ with high efficiency. It had thus been converted into phage $\mathbf{A}$.

Table 3 shows the result of growing all the adapted Vi-typing phages on type A. For the sake of completeness the ten typing phages examined by Anderson \& Felix $(1952,1953 a)$ are included. Phages B2 and B3 are omitted from Table 3 because their respective Vi-types have a low degree of specificity and show a wide range of sensitivity to many other adapted Vi-typing preparations (see Table 1). The specificity of phages B2 and B3 is difficult to distinguish from that of phage $A$, and satisfactory results could not be obtained in experiments carried out with them. Phage A is also omitted for obvious reasons.

The phages in Table 3 that had formerly been adapted to lysogenically determined Vi-types are starred. It can be seen that, of 13 phages showing the phenotypic change to phage $A, 12$ had been adapted to non-lysogenically determined types and one only (phage $\mathbf{T}$ ) to a lysogenically determined type. On the other hand, 5 out of the 11 phages which were propagated unchanged had been grown on lysogenically determined types; and 5 of the 6 phages changing in specificity but not yielding phage $\mathbf{A}$, being evidently phenotypic 
modifications of host-range mutants, had been propagated on lysogenically determined types. Thus, adaptation of Vi-phage II to non-lysogenically determined types appears to be mainly phenotypic in nature, whereas the

Table 2. Propagation of phage L2 on type $A$

\begin{tabular}{|c|c|c|c|c|c|}
\hline \multirow[b]{2}{*}{ Cultures } & \multirow{2}{*}{$\begin{array}{l}\text { Phage } \\
\text { dilutions }\end{array}$} & \multirow[b]{2}{*}{ Phage L2 } & \multicolumn{3}{|c|}{$\begin{array}{l}\text { Phage L2/A. } \\
\text { Preparations grown from } \\
\text { single plaques }\end{array}$} \\
\hline & & & Plaque 1 & Plaque 2 & Plaque 3 \\
\hline \multirow[t]{4}{*}{ Type A } & $\begin{array}{l}10^{-4} \\
10^{-5}\end{array}$ & $\begin{array}{l}\text { CL } \\
\text { CL }\end{array}$ & $\begin{array}{c}\mathrm{CL} \\
<\mathrm{CL}\end{array}$ & $\begin{array}{c}\mathrm{CL} \\
<\mathrm{CL}\end{array}$ & $\begin{array}{c}\mathrm{CL} \\
<\mathrm{CL}\end{array}$ \\
\hline & $10^{-6}$ & $<$ SCL & 58 & 93 & 6.5 \\
\hline & $10^{-7}$ & 37 & 18 & 13 & 8 \\
\hline & $10^{-8}$ & 1 & 2 & 1 & 2 \\
\hline \multirow[t]{5}{*}{ Type C } & $10^{-4}$ & 7 & 1 & - & - \\
\hline & $10^{-5}$ & - & - & - & --- \\
\hline & $10^{-6}$ & - & $\ldots$ & - & $\ldots$ \\
\hline & $10^{-7}$ & - & - & - & -- \\
\hline & $10^{-8}$ & - & - & - & - \\
\hline \multirow[t]{5}{*}{ Type E1 } & $10^{-4}$ & - & - & - & - \\
\hline & $10^{-5}$ & - & - & - & - \\
\hline & $10^{-6}$ & - & - & - & - \\
\hline & $10^{-7}$ & - & - & - & - \\
\hline & $10^{-8}$ & - & - & - & -- \\
\hline \multirow[t]{5}{*}{ Type F1 } & $10^{-4}$ & - & - & - & - \\
\hline & $10^{-5}$ & - & - & - & - \\
\hline & $10^{-6}$ & - & - & - & - \\
\hline & $10^{-7}$ & - & - & - & $\ldots$ \\
\hline & $10^{-8}$ & - & - & -- & - \\
\hline \multirow[t]{5}{*}{ Type J } & $10^{-4}$ & - & - & - & - \\
\hline & $10^{-5}$ & - & - & - & - \\
\hline & $10^{-6}$ & - & - & - & - \\
\hline & $10^{-7}$ &.- & - & $\ldots$ & ..- \\
\hline & $10^{-8}$ & - & - & - & -- \\
\hline \multirow[t]{5}{*}{ Type L1 } & $10^{-4}$ & CL & 2 & - & -- \\
\hline & $10^{-5}$ & CL & - & - & - \\
\hline & $10^{-6}$ & SCL & - & - & -- \\
\hline & $10^{-7}$ & 23 & - & - & - \\
\hline & $10^{-8}$ & 2 & - & - & - \\
\hline \multirow[t]{5}{*}{ Type L2 } & $10^{-4}$ & CL & 1 & 3 & 1 \\
\hline & $10^{-5}$ & SCL & -- & - & -- \\
\hline & $10^{-6}$ & +++ & - & - & $\ldots$ \\
\hline & $10^{-7}$ & 35 & - & - & $\ldots$ \\
\hline & $10^{-8}$ & 3 & - & - & - \\
\hline
\end{tabular}

$\mathrm{CL}=$ confluent lysis; $\mathrm{SCL}=$ semi-confluent lysis; $\angle \mathrm{CL}$ and $\angle \mathrm{SCL}=$ intermediate degrees of lysis; $+++=$ more than 150 plaques; $1,2,3$, etc. $=$ number of discrete plaques.

changes in host range of this phage during propagation on lysogenically determined types are in most instances partly or wholly genotypic. The further examination of the types not yet shown to be under lysogenic control that elicit a genotypic change in Vi-phage II has not so far been carried out.

Attention was concentrated on the phages that had undergone a change suggesting that they were phenotypic modifications of host-range mutants. 
These are shown in the third column of Table 3. They are listed separately in Table 4, together with the change in specificity they sustained after passage on type $\mathbf{A}$, and the determining phages carried by the types on which they had originally been grown. As phage D2 had been adapted to an apparently non-lysogenic type, it is not considered in the succeeding discussion.

Table 3. Results of propagation of specific Vi-typing phages on type A

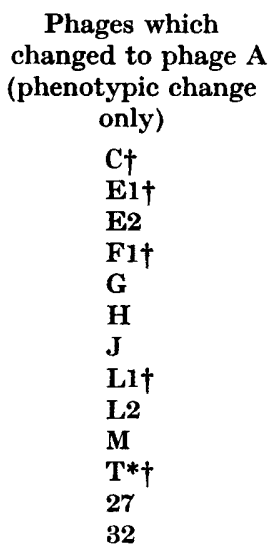

\begin{tabular}{|c|}
\hline $\begin{array}{l}\text { Phages propagated } \\
\text { unchanged } \\
\text { (host-range mutants }\end{array}$ \\
\hline B1 \\
\hline D1* \\
\hline D5† \\
\hline $\mathrm{D}^{* \dagger} \dagger$ \\
\hline $\mathbf{K} \ddagger$ \\
\hline $\mathrm{Nt}$ \\
\hline $\mathrm{O} \dagger$ \\
\hline $25^{*}$ \\
\hline $26^{*}$ \\
\hline $28 \ddagger$ \\
\hline $29^{*}$ \\
\hline E \\
\hline
\end{tabular}

Phages undergoing a change but not to phage A (phenotypically modified mutants)

* Phages corresponding to lysogenically determined types.

$\dagger$ Previously examined by Anderson \& Felix (1952, 1953 $a$ ).

$¥$ Types $K$ and 28 are lysogenic but their temperate phages do not appear to be concerned with type determination.

Table 4. Vi-typing phages undergoing modification when grown on type $A$ but not reverting to phage $A$

$\begin{array}{ccc} & & \begin{array}{c}\text { Determining } \\ \text { carried by } \\ \text { on which star } \\ \text { phage has bee }\end{array} \\ \text { Starting phage } & \text { Resulting phage } & (3) \\ \text { (1) } & (2) & - \\ \text { D2 } & \text { D1 } & \text { d1 } \\ \text { D4 } & \text { D1 } & \text { f2 } \\ \text { F2 } & 29 & \text { f2 } \\ \text { 30 } & 29 & 26^{\prime} \\ 31 & 26 & \text { d6 } \\ 33 & \text { D6 } & \end{array}$

The specificities of the phages in column 2 of Table 4 that had been originally adapted to lysogenic Vi-types are exactly those that would result from the growth of phage A on types D1, 29, 26 and D6 respectively. These types can be prepared from type $A$ by lysogenizing it with phages $d 1, f 2,26^{\prime}$ and $d 6$. We may now consider the types corresponding to the phages in column 1 of Table 4. The non-lysogenic type from which type $\mathrm{D} 4$ can be prepared is not known, but type F2 can be prepared from type F1 by lysogenizing it with phage $\mathrm{f} 2$; type 30 from type $\mathrm{C}$ with phage $\mathbf{2}$; type 31 from type $\mathbf{E} 1$ with 
phage $26^{\prime}$; and type 33 from type $\mathrm{C}$ with phage $\mathrm{d} 6$. Reference to Table 3 will show that the phages adapted to types F1, C and El are phenotypically modified, and all change back to phage $A$ when grown on type $A$. The same change had manifestly occurred in phages F 2, 30, 31 and 33, but reversion of their host ranges to that of phage $A$ was impossible because each adapted phage had already undergone the genotypic changes necessary to overcome the resistance conferred on the original host cells by their temperate determining phages. Thus, the end result of the growth of the Vi phages in column 1 of Table 4 on type A was to produce phages with specificities suggesting that they had been grown on a series of type $\mathbf{A}$ cultures lysogenized with type-determining phages $\mathrm{f} 2, \mathbf{2 6}^{\prime}$ and $\mathrm{d} 6$. Although the non-lysogenic precursor of type $\mathrm{D} 4$ is unknown, the change in phage $\mathrm{D}_{4}$ was clearly of the same nature. It is evident, therefore, that the effect of growth in type $\mathbf{A}$ cells on the specificity of the phages in column 1 was restricted to that portion of the phages that had been modified by the non-lysogenic component of each lysogenic complex on which they had originally been grown.

\section{The structural formulae of the Vi-types}

The information provided by the fact that naturally occurring Vi-types can be replicated in the laboratory by lysogenizing non-lysogenic types with suitable type-determining phages enables the lysogenically determined types to be considered on a structural basis. Adopting the current convention of designating a lysogenic strain by the symbol of the host cell followed by that of the carried phage in parenthesis, a series of structural formulae have been evolved for the Vi-types that owe their specificity at least in part to lysogenesis. The use of such formulae was first suggested by Luria (1953). The formulae used by the present writers are shown in Table 5 ; these give information con-

Table 5. The structural formulae of the lysogenically determined Vi-types of Salmonella typhi

\section{Original designation of Vi-type}

(1)

D1
D4
D6
F2
T
25 (Lie Kian Joe)
26 (Clark)
29 (Borman)
30 (Anderson)
31 (Wilson \& Edwards)
33 (Desranleau C2)

Type-

$\begin{array}{cc}\text { determining } & \text { Structural formula } \\ \text { phage carried } & \text { of Vi-type }\end{array}$

(2)

d1
d1
d6
f2
t
$25^{\prime}$
$26^{\prime}$
f2 or $30^{\prime}$
f2 or $30^{\prime}$
$26^{\prime}$
d6

(3)

$A(d 1)$
$?(d 1)^{*}$
$A(d 6) \dagger$
$F 1(f 2)$
$A(t)$
$A\left(25^{\prime}\right)$
$A\left(26^{\prime}\right)$
$A(f 2)$ or $A\left(30^{\prime}\right) \dagger$
$C(f 2)$ or $C\left(30^{\prime}\right) \dagger$
$E 1\left(26^{\prime}\right)$
$C(d 6)$

Types fully lysed by homologous adaptation of Vi-phage II in routine test dilution (4)
A, D1
$\mathrm{A}, \mathrm{D} 1, \mathrm{D} 4$
A, D6, 29
A, F1, F2, 29
A, $\mathbf{T}$
A, 25
A, 26
A, 29
A, C, 29, 30
A, E1, 26, 31
A, C, D6, 29, 30, 33

* The non-lysogenic precursor of type D4 is at present unknown.

$\uparrow$ Phages $\mathrm{d6}$, f2 and $30^{\prime}$ belong to a single serological group; they are also closely related in type-determining properties. 
cerning the phylogenetic relationships of the Vi-types concerned and also make it possible to predict the host ranges of the Vi-phage II preparations adapted to them. For example, type $\mathrm{D} 6$ is structurally $\mathrm{A}(\mathrm{d} 6)$ and type 33 is $\mathrm{C}(\mathrm{d} 6)$. When Vi-phage $\mathrm{II}$ is adapted to $\mathrm{C}(\mathrm{d} 6)$ it acquires simultaneously the lytic range of phage $\mathrm{C}$ and that of phage $\mathrm{D} 6$. Such an adaptation, therefore, will lyse type $\mathbf{A}$ (which is sensitive to all Vi-phage II adaptations), type $\mathrm{C}$, and also all lysogenic types descended from these two non-lysogenic precursors by lysogenization with phage $\mathrm{d} 6$ or a phage closely related to it. Phages $\mathrm{f} 2$ and $30^{\prime}$ are closely related to phage $\mathrm{d} 6$. Thus, the additional types lysed by phage 33 are: D6 $(=\mathrm{A}(\mathrm{d} 6)) ; \mathbf{2 9}\left(=\mathrm{A}(\mathrm{f} 2)\right.$ or $\left.\mathrm{A}\left(30^{\prime}\right)\right) ; 30\left(=\mathrm{C}(\mathrm{f} 2)\right.$ or $\left.\mathrm{C}\left(30^{\prime}\right)\right)$; and $33(=\mathrm{C}(\mathrm{d} 6))$ on which the phage was propagated. It must be pointed out here that, although phages f 2 and $30^{\prime}$ do not confer on a strain the property of resistance to phage $\mathrm{D} 6$, carriage of phage $\mathrm{d} 6$ endows cells with relative or complete resistance to Vi-phage II adapted to strains carrying phages $\mathrm{f} 2$ or $30^{\prime}$. Indeed, this resistance is the only known distinguishing feature between phages $\mathrm{d} 6$ and $\mathrm{f} 2$. Strains lysogenized with phages $\mathrm{f} 2$ and $\mathbf{3 0}^{\prime}$ show identical resistance patterns to the Vi-phage II typing preparations. They also select the same host-range mutant of Vi-phage II when the latter is grown on them. Phage f 2 is distinct from phage $\mathbf{3 0}^{\prime}$, however, because the former makes the strains it lysogenizes resistant to Craigie and Yen's Vi-phage IV whereas the latter does not. The determining phage of type 29 was formerly designated as phage 29' (Anderson \& Felix, 1953 $b$ ). Later work, however, has shown that this phage is indistinguishable from phage $\mathrm{f} 2$ and it is no longer considered by the present writers to merit separate recognition.

By a similar approach to that outlined for phage 33, the host range of phage 31 can be predicted. Type 31 is $\mathrm{E} 1\left(26^{\prime}\right)$. Vi-phage II adapted to it has the lytic range of phage $\mathrm{E} 1$ plus that of phage 26. The Vi-types lysed by phage 31 are, therefore: type $A$; type $\mathbf{E} 1$; and any lysogenic types carrying phage $26^{\prime}$ that are descended from these two precursors. Such types are type $26\left(=\mathrm{A}\left(26^{\prime}\right)\right)$ and type $31\left(=\mathrm{E} 1\left(26^{\prime}\right)\right)$ on which the phage was grown.

A lysogenically determined type is not sensitive to Vi-phage II adapted to another type carrying the same or a similar type-determining phage unless the non-lysogenic component of the first type is the same as that of the second or is type $A$, which is sensitive to all adaptations of Vi-phage II. Thus, type F 2, which is structurally $F 1(f 2)$, is resistant to phages 29 and 30 , both adapted to types carrying phage $\mathbf{f} 2$ or a phage very closely related to it (see Table 4). Phages F2, 29 and 30 all exhibit the same permanent genotypic change resulting from their being grown on strains carrying phage $\mathbf{2}$. In considering the relations of the specificities of these phages to one another, therefore, this permanent alteration can be disregarded and the differences between phages F2, 29 and 30 become identical with those between adaptations of Vi-phage II to types F1, A and C, which are the non-lysogenic precursors of types $\mathrm{F} 2(=\mathrm{F} 1(\mathrm{f} 2)), 29(=\mathrm{A}(\mathrm{f} 2))$ and $30(=\mathrm{C}(\mathrm{f} 2))$ respectively. Neither phage $A$ nor phage $\mathrm{C}$ will lyse type $\mathrm{F} 1$; therefore, neither phage 29 nor phage 30 will lyse type $\mathbf{F} 2$.

The host ranges of the adapted phages shown in column 4 of Table 5 have 
been restricted to parental non-lysogenic types and types carrying phages indistinguishable from or closely related to the phage determining the Vi-type to which Vi-phage II has been adapted. There are, however, other types, some lysogenic, others non-lysogenic, that are almost as sensitive to some heterologous adapted phages as they are to their homologous typing preparations. Types D1 and D5 are examples of this phenomenon. Both of these types are sensitive to phage D6. This is not shown in Table 5 but is in Table 1 . In the case of type $\mathrm{D} 1$, this sensitivity can be otherwise stated as indicating that lysogenization of type A with phage $d 1$ will not equip the cell with resistance against the host-range mutant of Vi-phage II that can lyse type D6. The reason for the phage sensitivity pattern of type $\mathrm{D5}$, which seems to be nonlysogenic, is not yet known, but its sensitivity to phage $\mathrm{D} 6$ is quite clear-cut. If a culture is sensitive to phage D6, it will be sensitive to adaptations of $\mathrm{Vi}$-phage II to any other types carrying phage d6. This is because type D6 is structurally $\mathrm{A}(\mathrm{d} 6)$ and in adaptation to this type Vi-phage II undergoes two changes: a mutation that enables it to overcome the block in its multiplication produced by the presence of phage $\mathrm{d} 6$ in the cell; and a phenotypic conditioning identical with that of phage $\mathbf{A}$ which has the narrowest of all host ranges. Type 33 is $\mathrm{C}(\mathrm{d} 6)$ : adaptation of $\mathrm{Vi}$-phage II to it demands the same host-range mutation as that present in phage $\mathrm{D} 6$ and a phenotypic modification identical with that of phage $\mathrm{C}$. The mutation common to phages $\mathrm{D} 6$ and 33 can be ignored when comparing their host ranges. The difference between the two phages then becomes identical with that between phage $\mathbf{A}$ and phage $\mathbf{C}$. Any strain attacked by phage $\mathbf{A}$ will be sensitive to phage $\mathbf{C}$; therefore, any strain lysed by phage $\mathrm{D} 6$ should be lysed by phage 33. This hypothesis is supported by the fact that types D1 and D5, which are sensitive to phage D6, are also sensitive to phage 33. Applying the same arguments, types D1 and D5 should be sensitive to Vi-phage II adapted to any other type carrying phage d6, irrespective of the non-lysogenic precursor of the type. An unnamed new type, identified in this laboratory in 1952, is at present known as T4904; it has the structural formula E1(d6). Phage A could not be adapted to this type, but phage $\mathbf{D} 6$ grew on it with ease to produce a phage which attacked types A, E1, D6 and T4904 with equal facility. As shown above, this phage should also be able to lyse types D1 and D5 and it does so.

\section{The independence of the phenotypic and genotypic changes in Vi-phage II}

A study of the formulae of the lysogenically determined types suggested that the adaptation of Vi-phage II to them consisted of the two separate processes of phenotypic modification and genotypic change, and it was decided to examine the extent to which these were independent of one another. Phages adapted to a selection of the lysogenically determined types were grown on non-lysogenic types known to be the precursors of other lysogenically determined complexes. Strain T4904 $(=\mathrm{El}(\mathrm{d} 6))$, referred to earlier, was included in these experiments. Two types prepared artificially in the laboratory 
were also included. One of these was $\mathrm{E} 1(\mathbf{f} 2)$ which was prepared by one of us (E.S.A.) in 1951. This has been referred to previously as ' a new subtype of the E group' (Felix \& Anderson, 1951; Anderson \& Felix, 1953b). Since these experiments were performed this type has been discovered in the field by Dr R. T. Scholtens (1955). We have examined the culture discovered by Scholtens, and find its formula to be $\operatorname{E1}\left(30^{\prime}\right)$. As has been indicated (see Table 5), types carrying phages $\mathbf{f} 2$ or $30^{\prime}$ present an identical lytic pattern with the adaptations of Vi-phage II. This type has been provisionally designated type E7. The second artificially prepared type used in these experiments was $\mathrm{EI}(\mathrm{d} 1)$ which is a further subtype of the $\mathbf{E}$ group.

In each case the non-lysogenic Vi-type chosen as the new host was known to be the precursor of a lysogenically determined type carrying a phage indistinguishable from that carried by the type on which the starting phage had originally been grown. For example, phage F2 was originally adapted to type F2, which is structurally F1(f2). Phage F2 was grown on type A, which is the non-lysogenic precursor of Type $29(=\mathbf{A}(\mathbf{f} 2))$. Since the change in Vi-phage II that enables it to overcome the resistance conferred on the cell by the presence of phage $\mathbf{f} 2$ is genotypic in nature and therefore stable, it was expected that the only change that would occur in phage F2 as the result of growth on type $\mathbf{A}$ would be that affecting the phenotypically controlled F 1 specificity which should change to that of phage $\mathbf{A}$. Thus, the specificity of phage F2 should change under these conditions from that corresponding to the F1(f2) complex to that corresponding to the $A(f 2)$ complex and the resulting phage should therefore be identical with phage 29 prepared by growing phage $\mathbf{A}$ on type 29. This prediction was confirmed and the principle was successfully applied to a number of other Vi-phage II typing preparations in which both phenotypic and genotypic changes were known to be present. These experiments are summarized in Table 6. The host ranges of the new phages shown in the fourth column of Table 6 were found to be identical with those of Vi-phage II preparations grown on the lysogenic types listed in the last column. All these host ranges had been predicted before the respective experiments were carried out.

These findings show that the phenotypic and genotypic changes in Vi-phage II are two entirely independent processes. Each host-range mutant as far as it was tested showed a phenotypic plasticity similar to that of phage A. The experimental results also prove the validity of the suggested structural formulae for the lysogenically determined Vi-types.

\section{DISCUSSION}

It was shown in earlier work (Anderson \& Felix, 1952, 1953a,c) that the changes in host range that occur in Vi-phage II when it is adapted to the various Vi-types of Salmonella typhi could be either phenotypic or genotypic in nature. In one instance, that of phage $\mathrm{D} 4$, both changes appeared to be represented. These observations were carried out on a limited number of Vi-typing preparations. The exploration of the complete range of Vi-typing 
phages has yielded the interesting result that most of the phenotypically changed preparations have been adapted to non-lysogenically determined Vi-types, whereas adaptation of Vi-phage II to lysogenically determined types usually requires genotypic or both genotypic and phenotypic changes. It is quite clear from the present series of experiments that the two alternative types of modification are independent of each other: it seems probable that they take place at different sites in Vi-phage II and concern different steps in the multiplication cycle of the phage.

Table 6. The adaptation to non-lysogenic types of Vi-typing phages

\begin{tabular}{|c|c|c|c|c|}
\hline \multicolumn{5}{|c|}{ originally grown on lysogenic types } \\
\hline $\begin{array}{l}\text { Starting } \\
\text { phage }\end{array}$ & $\begin{array}{l}\text { Structural } \\
\text { formula of } \\
\text { lysogenic type } \\
\text { on which starting } \\
\text { phage was grown }\end{array}$ & $\begin{array}{l}\text { Non-lysogenic } \\
\text { type on which } \\
\text { starting phage } \\
\text { is propagated }\end{array}$ & $\begin{array}{c}\text { Type } \\
\text { specificity } \\
\text { of resulting } \\
\text { phage }\end{array}$ & $\begin{array}{l}\text { Structural formula of } \\
\text { type to which new } \\
\text { phage corresponds }\end{array}$ \\
\hline D6 & $A(d 6)$ & $\mathbf{C}$ & 33 & $\mathrm{C}(\mathrm{d} 6)=$ type 33 \\
\hline D6 & $A(d 6)$ & E1 & 4904 & $\mathrm{E} 1(\mathrm{~d} 6)=\mathrm{T} 4904 *$ \\
\hline 33 & $\mathrm{C}(\mathrm{d} 6)$ & $\mathbf{A}$ & D6 & $A(d 6)=$ type $D 6$ \\
\hline 33 & $C(d 6)$ & E1 & 4904 & $\operatorname{El}(\mathrm{d} 6)=\mathrm{T} 4904$ \\
\hline F2 & F1(f2) & $\mathbf{A}$ & 29 & $A(f 2)=$ type 29 \\
\hline F2 & F1(f2) & C & 30 & $C(f 2)=$ type 30 \\
\hline 29 & $A(\mathrm{f} 2)$ & C & $\mathbf{3 0}$ & $\mathrm{C}(\mathrm{f} 2)=$ type 30 \\
\hline 30 & $\mathrm{C}(\mathrm{f} 2)$ & A & 29 & $\mathrm{~A}(\mathrm{f} 2)=$ type 29 \\
\hline 26 & $\mathbf{A}\left(26^{\prime}\right)$ & E1 & 31 & $\operatorname{E1}\left(26^{\prime}\right)=$ type 31 \\
\hline $\mathbf{3 1}$ & E1 $\left(26^{\prime}\right)$ & A & 26 & $A\left(26^{\prime}\right)=$ type 26 \\
\hline D1 & $A(d 1)$ & E1 & E1(d1) & $\mathrm{E} 1(\mathrm{~d} 1) \dagger$ \\
\hline F2 & Fi(f2) & E1 & E1(f2) & E1(f2) $\dagger$ \\
\hline
\end{tabular}

Phenotypic modification affecting phages was first reported by Luria \& Human (1952) in phage T2. Bertani \& Weigle (1953) and Weigle \& Bertani (1953) studied phenotypic changes in phage $\mathrm{P2}$, a temperate phage isolated from Escherichia coli by Lisbonne \& Carrère, and $\lambda$, the temperate phage carried by $\boldsymbol{E}$. coli K-12. Fluctuation tests carried out by the latter workers with single cycles of phage multiplication showed that the number of plaques produced by a phage on a new culture, to which it could be phenotypically adapted but on which only a small fraction of the viable particles would initially form plaques, conformed to a Poisson distribution. From this they concluded that the particles undergoing adaptation to the new host were not produced by spontaneous mutation in the original stock of phage. Other experiments performed by these workers suggested that the potentiality to produce the altered host range in the phage was a property of exceptional cells of the new host and not of exceptional particles of the phage. The subject of phenotypic (or 'host induced') modification in phage has been reviewed by Luria (1953).

For the unequivocal demonstration that a change of host range in phage is purely phenotypic in nature it is necessary to show that it is completed in a single cycle of phage growth in the host cells. The work reported here, although 
performed in all cases with single-plaque lines of phage, was not checked by single-cycle experiments. Nevertheless, because of the uniformity of host range of all phage lines descended from single plaques picked from the growth on type $\mathbf{A}$ of any typing phage which has been designated as a phenotypic or partly phenotypic modification of Vi-phage II, it seemed reasonable to regard such preparations as having undergone a phenotypic change only. We have recently carried out single-cycle experiments which justify this assumption and similar observations have been made by Baron, Formal \& Spilman (1955). We have also shown that the genotypically changed preparations are descended from selected host-range mutants that occur in concentrated preparations of phage $\mathbf{A}$ and that the mutations are independent of the presence of the heterologous bacterial types. An account of this work will be given in a later publication.

The use of the structural formulae proposed for the lysogenically determined types offers considerable advantages over the single-symbol designations in current use in the study of the factors controlling Vi-type specificity. As we have pointed out earlier, these formulae show the phylogenetic relationships of the lysogenic types. These types can be regarded as consisting of two separate components: one is the non-lysogenic ancestor of the type, which produces a phenotypic change only in Vi-phage II; and the other is the temperate phage which, in virtue of its association with the nuclear apparatus of the cell, is able to effect a block in the multiplication of the wild genotype of Vi-phage II. This block can only be by-passed or otherwise overcome by hostrange mutants of the Vi phage. The site or factor in the cell which controls the phenotypic modification of the Vi phage is independent of, and unaffected by, lysogenization by type-determining phages. It can be regarded as a permanent characteristic of the cell that may be masked but not changed by the attainment of the lysogenic state. An apparent exception to this rule is found in type $\mathbf{T}$, which is structurally $\mathbf{A}(t)$ (see Table 5 ): in this case the determining phage seems to induce only a phenotypic change in Vi-phage II.

The structural formulae show what phenotypic modifications are superimposed on particular mutants in the preparation of some of the Vi-typing phages and make it possible to predict the host ranges of the respective adaptations. Moreover, the formulae indicate the host-range mutant that can be grown on a lysogenically determined type if a typing phage of reasonable titre is difficult or impossible to prepare by propagating phage $\mathbf{A}$ on it. For example, it is rather difficult to grow phage $A$ on type $33(=C(d 6)$; see Table 5) so as to produce a high-titre typing preparation in a single manipulation. On the other hand, phage D6 (a host-range mutant) will grow quite easily on type 33 to produce high-titre typing preparations which are indistinguishable in specificity from those prepared by the more conventional procedure. In fact, as has been shown (see Table 6) a typing phage of identical specificity can be prepared by growing phage $\mathrm{D} 6$ on type $\mathrm{C}$.

It is the present custom to group Vi-types solely according to their reactions with the adapted typing preparations. The lysogenically determined types, however, could be grouped according to the non-lysogenic types from which 
they are derived and the phages they carry. As these two factors control the reactions of a strain with the typing phages, they each present a basis for classification. In Table 7 the types concerned are grouped on the basis of common non-lysogenic ancestry, the 'parent' type being placed at the head of each group. A number of non-lysogenic types exist that are related to type $\mathbf{E} 1$ in a way that is at present obscure. These types are mostly sensitive only to their adapted Vi-phages but these phages will also readily lyse type $\mathbf{E} 1$. Type E 2 is an example of this phenomenon (see Table 1); and other instances have been discovered by various workers. These types are omitted from Table 7, which is concerned only with the relations of non-lysogenic precursors

Table 7. A classification of Vi-types of Salmonella typhi by their structural formulae

\begin{tabular}{|c|c|c|}
\hline Group & Original designation & Formula \\
\hline $\mathbf{A}$ & $\begin{array}{l}\text { A } \\
\text { D1 } \\
\text { D6 } \\
29 \text { (Borman) } \\
\text { T } \\
25 \text { (Lie Kian Joe) } \\
26 \text { (Clark) }\end{array}$ & $\begin{array}{l}\mathbf{A} \\
\mathbf{A}(\mathrm{d} 1) \\
\mathbf{A}(\mathrm{d} 6)^{*} \\
\mathbf{A}(\mathrm{f} 2) \text { or } \mathbf{A}\left(30^{\prime}\right)^{*} \\
\mathbf{A}(\mathrm{t}) \\
\mathbf{A}\left(25^{\prime}\right) \\
\mathbf{A}\left(26^{\prime}\right)\end{array}$ \\
\hline C & $\begin{array}{l}\mathrm{C} \\
30 \text { (Anderson) } \\
33 \text { (Desranleau C2) }\end{array}$ & $\begin{array}{l}\mathrm{C} \\
\mathrm{C}(\mathrm{f} 2) \text { or } \mathrm{C}\left(30^{\prime}\right) \\
\mathrm{C}(\mathrm{d} 6)\end{array}$ \\
\hline $\mathbf{E}$ & $\begin{array}{l}\text { E1 } \\
\text { T4904 } \\
31 \text { (Wilson \& Edwards) } \\
\text { Artificially prepared types }\end{array}$ & $\begin{array}{l}\text { E1 } \\
\text { E1(d6) } \\
\text { E1(26') } \\
\left\{\begin{array}{l}\text { E1(d1) } \\
\text { E1(f2) }\end{array}\right.\end{array}$ \\
\hline $\mathbf{F}$ & $\begin{array}{l}\text { F1 } \\
\text { F2 }\end{array}$ & $\begin{array}{l}\text { F1 } \\
\text { F1(f2) }\end{array}$ \\
\hline
\end{tabular}

* See footnote to Table 5 concerning phages d6, f2 and $30^{\prime}$.

to lysogenically determined types of which the structural formulae are known. Type D4, which carries phage $d 1$, cannot at present be inserted into this scheme because its non-lysogenic precursor is unknown. New lysogenically determined Vi-types can be added to the scheme shown in Table 7 as they are discovered.

It would, of course, be equally possible to classify lysogenic strains according to the phages they carry, but subdivision on this basis would necessarily omit related non-lysogenic types and full use would not be made of the available information. The proposed nomenclature and supplementary method of classification clarify the interactions of the Vi-phage types and their respective typing phages. As we have stated earlier, experiments have been carried out to examine the statistical distribution of particles undergoing phenotypic and genotypic changes. These form the subject of a separate communication which will be published in the near future. 


\section{REFERENCES}

Anderson, E. S. (1951). The significance of Vi-phage types F 1 and F2 of Salmonella typhi. J. Hyg., Camb. 49, 458.

Anderson, E. S. (1955). Consideration of the Vi-phage types of Salmonella typhi on a structural basis. Nature, Lond., 175, 171.

Anderson, E. S. \& Felix, A. (1952). Variation in Vi-phage II of Salmonella typhi. Nature, Lond., 170, 492.

Anderson, E. S. \& Felix, A. (1953a). 'Degraded Vi strains' and variation in Vi-phage II of Salmonella typhi. J. gen. Microbiol. 8, 408.

Anderson, E. S. \& Felix, A. (1953b). The Vi type-determining phages carried by Salmonella typhi. J. gen. Microbiol. 9, 65.

Anderson, E. S. \& Feurx, A. (1953c). Vi-phage-type specificity and degradation in Salmonella typhi. Proc. Congr. int. Microbiol. 6, Rome, 3, 462.

Baron, L. S., Format, S. B. \& Spilman, W. (1955). Vi phage-host interaction in Salmonella typhosa. J. Bact. 69, 177.

Bertani, G. \& Weigle, J. J. (1953). Host controlled variation in bacterial viruses. J. Bact. 65, 113.

Craigie, J. (1940). Variation of type II Vi phage. Proc. Congr. int. Microbiol. 3, New York. 296.

Craigie, J. \& Yen, C. H. (1938). The demonstration of types of B. typhosus by means of preparations of Type II Vi phage. Canad. publ. Hlth J. 29, 448, 484.

Felix, A. \& Anderson, E. S. (1951). Bacteriophages carried by the Vi-phage types of Salmonella typhi. Nature, Lond., 167, 603.

LurIA, S. E. (1953). Host-induced modifications of viruses. Cold Spr. Harb. Symp. quant. Biol. 18, 237.

LuriA, S. E. \& Human, M. L. (1952). A non-hereditary, host-induced variation of bacterial viruses. J. Bact. 64, 557.

Scholtens, R. T. (1955). Vi-phage typing of Salmonella typhi. Extension of Vitype group $\mathrm{E}$ and observations on group ' 29 '. Ann. Inst. Pasteur, 89, 216.

Weigle, J. J. \& Bertani, G. (1953). Variations des bactériophages conditionnées par les bactéries hôtes. Ann. Inst. Pasteur, 84, 175. 